\title{
Spatio-kinematical model of the collimated molecular outflow in the water-fountain nebula IRAS 16342-3814^
}

\author{
D. Tafoya (多穂谷 $)^{1,2}$, G. Orosz ${ }^{3,4}$, W. H. T. Vlemmings ${ }^{2}$, R. Sahai ${ }^{5}$, and A. F. Pérez-Sánchez ${ }^{6}$ \\ ${ }^{1}$ Chile Observatory, National Astronomical Observatory of Japan, National Institutes of Natural Sciences, 2-21-1 Osawa, \\ Mitaka, Tokyo 181-8588, Japan \\ ${ }^{2}$ Department of Space, Earth and Environment, Chalmers University of Technology, Onsala Space Observatory, \\ 43992 Onsala, Sweden \\ e-mail: daniel.tafoya@chalmers.se \\ ${ }^{3}$ School of Natural Sciences, University of Tasmania, Private Bag 37, Hobart, Tasmania 7001, Australia \\ ${ }^{4}$ Xinjiang Astronomical Observatory, Chinese Academy of Sciences, 150 Science 1-Street, Urumqi, Xinjiang 830011, PR China \\ 5 Jet Propulsion Laboratory, MS 183-900, California Institute of Technology, Pasadena, CA 91109, USA \\ ${ }^{6}$ European Southern Observatory, Alonso de Córdova 3107, Vitacura, Casilla 19001, Santiago de Chile
}

Received 13 November 2018 / Accepted 13 June 2019

\begin{abstract}
Context. Water-fountain nebulae are asymptotic giant branch (AGB) and post-AGB objects that exhibit high-velocity outflows traced by water-maser emission. Their study is important for understanding the interaction between collimated jets and the circumstellar material that leads to the formation of bipolar and/or multi-polar morphologies in evolved stars.

Aims. The aim of this paper is to describe the three-dimensional morphology and kinematics of the molecular gas of the water-fountain nebula IRAS 16342-3814.

Methods. Data was retrieved from the ALMA archive for analysis using a simple spatio-kinematical model. The software SHAPE was employed to construct a three-dimensional, spatio-kinematical model of the molecular gas in IRAS 16342-3814, and to then reproduce the intensity distribution and position-velocity diagram of the CO emission from the ALMA observations to derive the morphology and velocity field of the gas. Data from $\operatorname{CO}(J=1 \rightarrow 0)$ supported the physical interpretation of the model.

Results. A spatio-kinematical model that includes a high-velocity collimated outflow embedded within material expanding at relatively lower velocity reproduces the images and position-velocity diagrams from the observations. The derived morphology is in good agreement with previous results from IR and water-maser emission observations. The high-velocity collimated outflow exhibits deceleration across its length, while the velocity of the surrounding component increases with distance. The morphology of the emitting region, the velocity field, and the mass of the gas as function of velocity are in excellent agreement with the properties predicted for a molecular outflow driven by a jet. The timescale of the molecular outflow is estimated to be $\sim 70-100 \mathrm{yr}$. The scalar momentum carried by the outflow is much larger than it can be provided by the radiation of the central star. An oscillating pattern was found associated with the high-velocity collimated outflow. The oscillation period of the pattern is $T \approx 60-90 \mathrm{yr}$ and its opening angle is $\theta_{\mathrm{op}} \approx 2^{\circ}$.

Conclusions. The $\mathrm{CO}(J=3 \rightarrow 2)$ emission in IRAS 16342-3814 is interpreted in terms of a jet-driven molecular outflow expanding along an elongated region. The position-velocity diagram and the mass spectrum reveal a feature due to entrained material that is associated with the driving jet. This feature is not seen in other more evolved objects that exhibit more developed bipolar morphologies. It is likely that the jet in those objects has already disappeared since it is expected to last only for a couple hundred years. This strengthens the idea that water fountain nebulae are undergoing a very short transition during which they develop the collimated outflows that shape the circumstellar envelopes. The oscillating pattern seen in the $\mathrm{CO}$ high-velocity outflow is interpreted as due to precession with a relatively small opening angle. The precession period is compatible with the period of the corkscrew pattern seen at IR wavelengths. We propose that the high-velocity molecular outflow traces the underlying primary jet that produces such a pattern.
\end{abstract}

Key words. stars: AGB and post-AGB - stars: jets - stars: mass-loss - stars: winds, outflows - submillimeter: stars

\section{Introduction}

There is a growing consensus that collimated jet-like outflows create cavities with dense walls in the slowly expanding circumstellar envelopes (CSE) of post-asymptotic giant branch (post-AGB) stars. Once the central star becomes hot enough to ionise the circumstellar material, such structures are seen as beautiful and colourful bipolar or even multi-polar planetary nebulae (PNe) (Sahai \& Trauger 1998). Water-fountain nebulae (wf-nebulae) are a reduced group of AGB and post-AGB objects

\footnotetext{
* The reduced datacube is only available at the CDS via anonymous ftp to cdsarc.u-strasbg. fr $(130.79 .128 .5)$ or via http://cdsarc. u-strasbg.fr/viz-bin/qcat?J/A+A/629/A8
}

that are thought to be experiencing the first manifestation of such collimated outflows (Imai 2007). Therefore these objects are one of the best tools that we have available to probe and study the mechanisms of jet launching and collimation in evolved stars, as well as the interaction of the jet with the CSE.

IRAS 16342-3814 was the first object to be classified as a wf-nebula given the wide diffusion of its $\mathrm{H}_{2} \mathrm{O}$ maser emission of $\sim 260 \mathrm{~km} \mathrm{~s}^{-1}$ (Likkel \& Morris 1988). IRAS 16342-3814 is one of the best studied wf-nebulae, observed over a wide range of wavelengths across the electromagnetic spectrum, from optical to radio (Sahai et al. 1999, 2005, 2017; Gledhill \& Forde 2012; Imai et al. 2012). The distribution and kinematics of the circumstellar material has also been studied via its $\mathrm{H}_{2} \mathrm{O}$ and $\mathrm{OH}$ maser emission (Claussen et al. 2009; Sahai et al. 1999). 
The Hubble Space Telescope (HST) and Keck Adaptive Optics (AO) images of IRAS 16342-3814 show a small (3") bipolar nebula, with the lobes separated by a dark, equatorial waist (Sahai et al. 1999, 2005). The morphology seen in the images implies that the lobes are bubble-like reflection nebulae illuminated by starlight escaping through polar holes in a dense, dusty waist obscuring the central star. The AO observations reveal a corkscrew-shaped pattern apparently etched into the lobe walls, which is inferred to be the signature of an underlying precessing jet. This jet has presumably carved out the observed bipolar cavities in the surrounding envelope formed during the AGB phase of the star (Sahai et al. 2005). Gledhill \& Forde (2012) studied the $\mathrm{H}_{2}$ emission of this source and concluded that it is likely that the jet and outflow are powered by accretion onto a binary companion. The $\mathrm{H}_{2} \mathrm{O}$ masers in IRAS 16342-3814 are thought to be tracing bow shocks at the ends of a jet. From the analysis of the proper motions of the masers, Claussen et al. (2009) derived an expansion velocity for the material in the head of the jet of $v_{\exp } \sim 155-180 \mathrm{~km} \mathrm{~s}^{-1}$.

Recently, Sahai et al. (2017) carried out observations of the molecular emission of IRAS 16342-3814 with ALMA. From the results of their observations these authors concluded that the emission originates in a precessing molecular outflow with a wide precession angle of $\sim 90^{\circ}$. However, it is difficult to explain the creation of an outflow with such a wide precession angle by the jet underlying the corkscrew pattern seen from the AO observations. In this work we present a different spatio-kinematical model of the collimated molecular outflow in the wf-nebula IRAS 16342-3814 that considers a small opening angle, which is in better agreement with the results from previous observations.

\section{CO data of IRAS 16342-3814 from ALMA}

We retrieved archive ALMA data of the project 2012.1.00678.S (PI: R. Sahai) to study in more detail the kinematics of the molecular gas in IRAS 16342-3814. The spectral setup, sensitivity, angular resolution, and results of the observations of the $\mathrm{CO}(J=3 \rightarrow 2),{ }^{13} \mathrm{CO}(J=3 \rightarrow 2)$, and $\mathrm{H}^{13} \mathrm{CO}(J=4 \rightarrow 3)$ emission lines were reported by Sahai et al. (2017). In this work we further analyse the kinematics of the molecular gas using the emission from the $\mathrm{CO}(J=3 \rightarrow 2)$ line, as this emission line has the highest signal-to-noise ratio. Since the position-velocity $(\mathrm{P}-\mathrm{V})$ diagrams of the ${ }^{13} \mathrm{CO}(J=3 \rightarrow 2)$ and $\mathrm{H}^{13} \mathrm{CO}(J=4 \rightarrow 3)$ are very similar to that of the $\mathrm{CO}(J=3 \rightarrow 2)$ line, the main results presented in this work are valid for the three lines.

In addition to the archival data, we have also obtained data from ALMA observations of the $\operatorname{CO}(J=1 \rightarrow 0)$ line. These observations are part of the project 2018.1.00250.S (PI: D. Tafoya) and were carried out on December 9, 2018 using 43-12 $\mathrm{m}$ antennas with maximum and minimum baselines of 740 and $15 \mathrm{~m}$, respectively. The resulting angular resolution was around $1^{\prime \prime}$ and the maximum recoverable scale was 11." 8 . The data were calibrated with the ALMA pipeline using the CASA version 5.4.0-68. Subsequently, the data were self-calibrated in phase following the standard self-calibration guidelines for ALMA data. In the present work, the observations of the $\mathrm{CO}(J=1 \rightarrow 0)$ line are used mainly to support the conclusions drawn from the $\mathrm{CO}(J=3 \rightarrow 2)$ emission (see Sect. 4), but these will be presented and discussed in more detail elsewhere.

From the data cube of the $\mathrm{CO}(J=3 \rightarrow 2)$ line we extracted the peak position of the brightness distribution in every channel within the velocity range $-235<v_{\mathrm{LSR}}\left(\mathrm{km} \mathrm{s}^{-1}\right)<+325$ using the task JMFIT of the Astronomical Image Processing System
(AIPS $)^{1}$. The emission within this velocity range corresponds to the high-velocity outflow (HVO) observed by Sahai et al. (2017), see their Figs. 1b and 2. It should be noted that apart from this emission, Sahai et al. (2017) also identified an extreme high-velocity outflow (EHVO) with higher expansion velocities. However, here and in the following sections we only consider the emission associated with the HVO and leave the discussion of the nature of the EHVO for Sect. 4.2. From the measured peak positions of the $\mathrm{CO}(J=3 \rightarrow 2)$ emission we estimated the centroid of the distribution by computing the non-weighted mean value. The position of the centroid was used as a reference position throughout the analysis described below. In Fig. 1a we plot the declination (Dec) and RA offsets of the emission peak positions with respect to the reference position. Subsequently, we calculated the separation of the emission peak positions to the reference position as Dist.offset $= \pm \sqrt{\text { RA offset }}{ }^{2}+$ Dec offset $^{2}$. We used the minus sign when RA offset was negative, otherwise we used the plus sign. In Fig. 1b we plot the distance offset of the emission peak position as a function of the velocity offset of the channel. The velocity offsets are defined with respect to the systemic velocity of the source, assumed to be $v_{\text {sys,LSR }}=45 \mathrm{~km} \mathrm{~s}^{-1}$ (Sahai et al. 2017). The distribution of the points in the plot of Fig. 1b exhibits an S-like shape similarly to the emission in the P-V diagram obtained by Sahai et al. (2017, see their Fig. 2). This is due to the fact that the plot shown in Fig. $1 \mathrm{~b}$ is equivalent to a P-V diagram since the emission peak positions lie basically along one single direction on the sky (position angle, PA, $\sim 67^{\circ}$, see Fig. 1a).

From the plot of Fig. 1b it can be seen that for velocities $\left|v_{\text {offset }}\right|<150 \mathrm{~km} \mathrm{~s}^{-1}$ the absolute value of the velocity offset increases monotonically as a function of the absolute distance offset, $|\mathrm{d} v| /|\mathrm{d} r|>0$. For $\left|v_{\text {offset }}\right|>150 \mathrm{~km} \mathrm{~s}^{-1}$ the gradient is opposite, $|\mathrm{d} v| /|\mathrm{d} r|<0$. Therefore, this plot suggests the presence of different molecular gas components with different kinematical signatures. We will refer to these components as "kinematical components". In the following we consider these two types of kinematical components, one with positive velocity gradient and the other with negative velocity gradient. Surprisingly, as the plot of Fig. 1a shows, even though these components have different kinematical signatures, they are almost perfectly aligned on one single direction on the plane of the sky.

In order to visualise the spatial distribution of the emission of the kinematical components defined above, we created velocity-integrated images of the $\mathrm{CO}(J=3 \rightarrow 2)$ emission taking into account their corresponding velocity ranges. We considered three velocity ranges as follows: $-150<v_{\text {offset }}\left(\mathrm{km} \mathrm{s}^{-1}\right)<+150$, $-270<v_{\text {offset }}\left(\mathrm{km} \mathrm{s}^{-1}\right)<-150$, and $+150<v_{\text {offset }}\left(\mathrm{km} \mathrm{s}^{-1}\right)<+270$. The first velocity range corresponds to the kinematical component with positive velocity gradient while the last two correspond to the kinematical component with negative velocity gradient.

In Fig. 2 we show contour maps of the integrated $\mathrm{CO}(J=3 \rightarrow 2)$ emission obtained from the velocity ranges considered above. It should be noted that this colour coding is the same as the one for the kinematical components shown in Fig. 1. From Fig. 2 it can be seen that the emission with velocity-offsets $\left|v_{\text {offset }}\right|>150 \mathrm{~km} \mathrm{~s}^{-1}$, from now on referred to as high-velocity emission, traces a pair of collimated bipolar lobes along the direction with $\mathrm{PA}=\sim 67^{\circ}$. The velocity-integrated intensity of the high-velocity emission has two emission peaks located at

\footnotetext{
1 AIPS is produced and maintained by the National Radio Astronomy Observatory, a facility of the National Science Foundation operated under cooperative agreement by Associated Universities, Inc.
} 

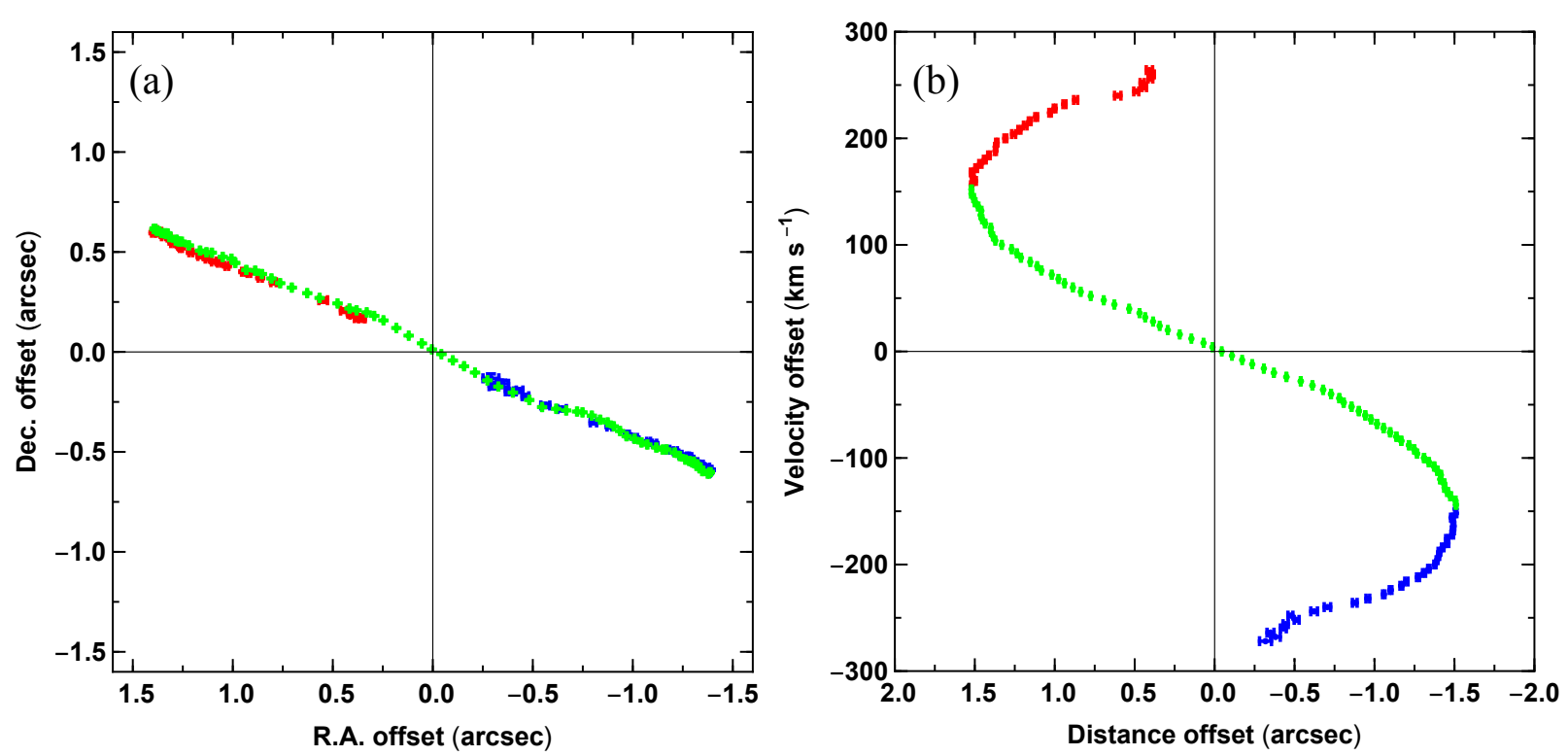

Fig. 1. Kinematical components of $\mathrm{CO}(3 \rightarrow 2)$ emission from IRAS 16342-3814. Panel a: peak positions of the brightness distribution of $\mathrm{CO}(3 \rightarrow 2)$ emission for each individual velocity channel obtained with the task JMFIT of AIPS. The origin corresponds to the non-weighted mean position of the distribution. The negative right ascension (RA) offsets are on the right side of the plot. Panel $b$ : distance offsets from the mean position as a function of the velocity offset from the systemic velocity $v_{\text {sys,LSR }}=45 \mathrm{~km} \mathrm{~s}^{-1}$. The colour of the data points are coded according to their velocity gradient, $|\mathrm{d} v| /|\mathrm{d} r|$ : green for $|\mathrm{d} v| /|\mathrm{d} r|>0$, and blue and red for $|\mathrm{d} v| /|\mathrm{d} r|<0$. The negative distance offsets correspond to data points with negative RA offsets and these are on the right side of the plot. The error bars of the positions are included in the plot but their size is similar to the size of the data points.

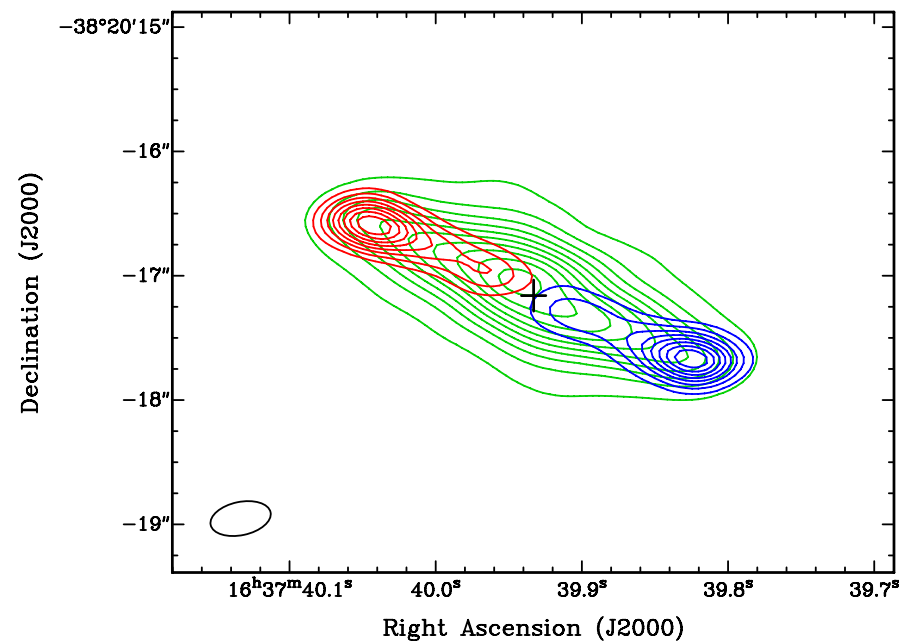

Fig. 2. Velocity-integrated $\mathrm{CO}(J=3 \rightarrow 2)$ emission of IRAS 16342-3814. The blue, green, and red contours correspond to emission at velocity ranges $-270<v_{\text {offset }}\left(\mathrm{km} \mathrm{s}^{-1}\right)<-150$, $-150<v_{\text {offset }}\left(\mathrm{km} \mathrm{s}^{-1}\right)<+150$, and $+150<v_{\text {offset }}\left(\mathrm{km} \mathrm{s}^{-1}\right)<+270$, respectively. The levels of the blue and red contours go from 20 to $90 \%$ the peak value of the emission (8.3 and $6.6 \mathrm{Jy} \mathrm{beam}^{-1} \mathrm{~km} \mathrm{~s}^{-1}$, respectively) at steps of $10 \%$. The levels of the green contours go from 5 to $95 \%$ the peak value of the emission $\left(50.7 \mathrm{Jy} \mathrm{beam}^{-1} \mathrm{~km} \mathrm{~s}^{-1}\right)$ at steps of $10 \%$. The rms value of the velocity-integrated maps is $\sim 7 \times 10^{-2} \mathrm{Jy}_{\text {beam }}^{-1} \mathrm{~km} \mathrm{~s}^{-1}$. The cross indicates the peak of the continuum emission. The ellipse located at the lower left corner indicates the synthesised beam, $\theta_{\mathrm{FWHM}}=0,{ }^{\prime \prime} 49 \times 0.0^{\prime \prime} 27$, position angle $(\mathrm{PA})=-78^{\circ}$.

the tips of the bipolar lobes. The geometric centre of the bipolar lobes coincides with the central part of the nebula, which is indicated with a cross that corresponds to the peak position of the continuum emission, (J2000) RA=16:37:39.935, Dec $=-38: 20: 17.15$. The structure traced by emission with velocity offsets $\left|v_{\text {offset }}\right|<150 \mathrm{~km} \mathrm{~s}^{-1}$, from now on referred to as "low-velocity emission", exhibits a more extended morphology encompassing the high-velocity emission. The low-velocity emission has only one intensity peak located near the centre of the system. The width of the brightness distribution of the lowvelocity emission decreases along the direction of the bipolar axis resembling an elongated diamond-like shape.

\section{Spatio-kinematical model of molecular gas}

In this section we present a simple spatio-kinematical model of the molecular gas of IRAS 16342-3814 to reproduce the spatial distribution and velocity gradients seen in Figs. 1 and 2. In the following we describe the considerations that we made to define the morphology and velocity field used in our model. First we note from Fig. 1a that the spatial distribution of the emission peak positions of the kinematical components, defined in Sect. 2, lie along one single direction on the plane of the sky. This could be due to the kinematical components being intrinsically aligned on one single direction, or alternatively they could lie on different directions but appear perfectly aligned on the plane of the sky. We consider that the latter possibility is very unlikely as even a small inclination of the system with respect to the line-of-sight would result in the components appearing misaligned on the plane of the sky. Furthermore, previous observations suggest that the gas and dust are located within a narrow cone, elongated along the direction with PA $\sim 67^{\circ}$ (Sahai et al. 2005; Claussen et al. 2009; Imai et al. 2012; Gledhill \& Forde 2012). Therefore, in this work we adopted a geometry similar to the one assumed in previous works and propose that the observed $\mathrm{CO}(J=3 \rightarrow 2)$ emission can be explained using two morphological structures associated with the kinematical components defined in Sect. 2. First, an elongated structure with a relatively wide waist and narrow tips. This component is associated with the low-velocity emission and we will refer to it 

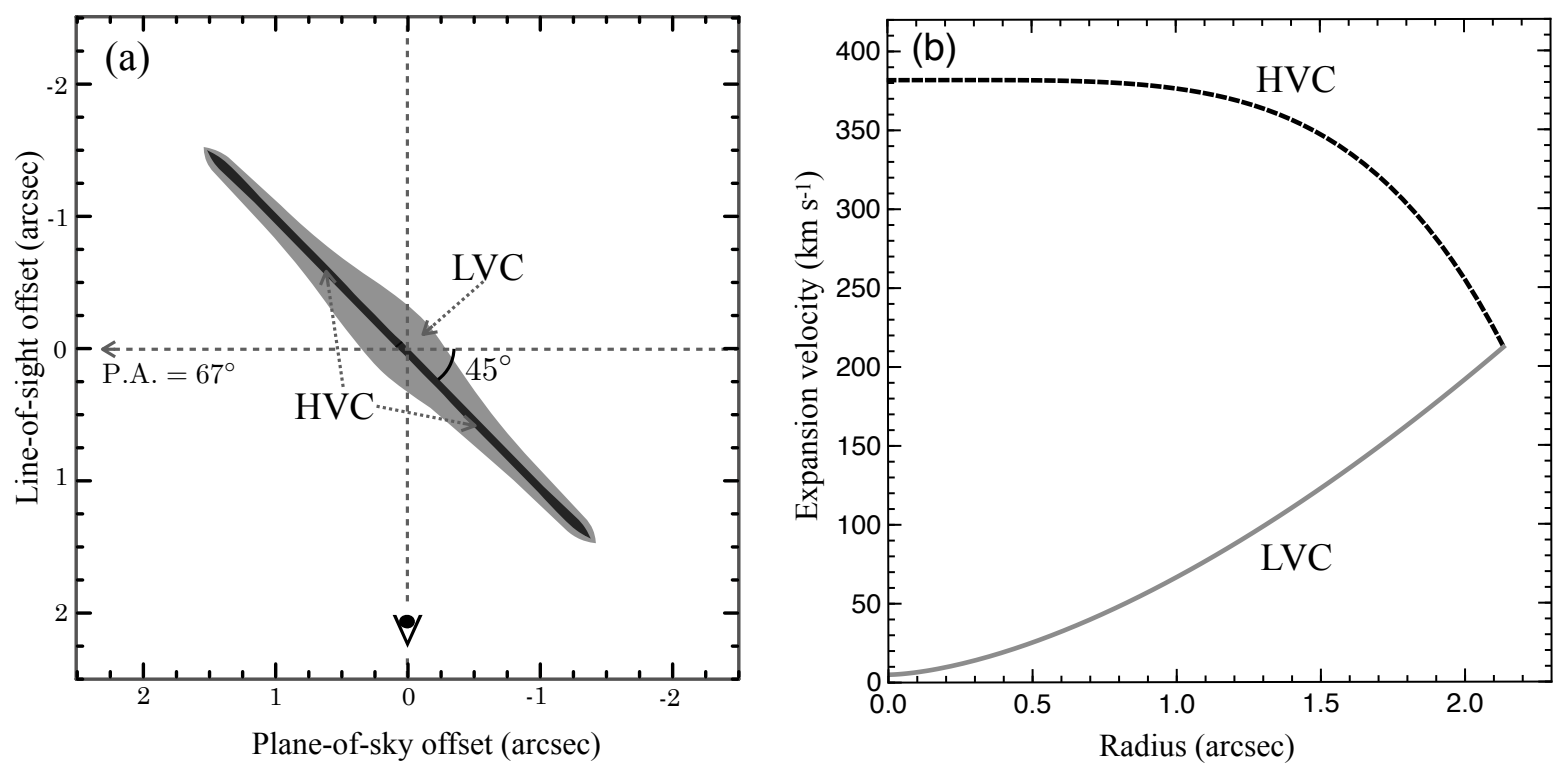

Fig. 3. Spatio-kinematical model of molecular gas of IRAS 16342-3814. Panel a: geometry of the spatio-kinematical model of the molecular envelope of IRAS 16342-3814. It should be noted that the $y$-axis is angular size along the line-of-sight and the $x$-axis is angular size on the plane of the sky. We assume an inclination of the major axis of the nebula of $45^{\circ}$ with respect to the plane of the sky (Claussen et al. 2009). The grey region indicates the LVC component. The black region indicates the HVC component. Panel $b$ : expansion velocity as a function of the separation from the center of the system. The grey and black lines are the velocity laws for the LVC and HVC components, respectively. The mathematical expression for the velocity laws is given in Eq. (1).

as the low-velocity component (LVC). The second assumption is a pencil-like, collimated structure associated with the highvelocity emission that lies embedded within the LVC component. We will refer to this component as the high-velocity component (HVC). Thus, the morphology for the CO emitting region of IRAS 16342-3814 in our spatio-kinematical model has a shape that resembles a French spindle, as it is shown in Fig. 3a.

The width of the LVC, as a function of the velocity, was determined by fitting a two-dimensional Gaussian function to the intensity distribution in each individual channel of the data cube. The size was set equal to the quadratic average of the major and minor axis, deconvolved from the beam, of the fitted elliptical Gaussian function. The width of this component, at its waist, is $\sim 0$.' 5 and it decreases monotonically to reach a value of $\sim 0$.' 15 at the polar tips. The width of the HVC is not resolved by these observations but we adopted a width at the equator of $\sim 0$.' 02 and set it to increase monotonically to a value of $\sim 0$." 05 based on the opening angle of the sinusoidal distribution of the peak positions of this component (see Sect. 4.2 and Fig. 8). The major axis of both components was assumed to have an inclination angle with respect to the plane of the sky $\theta_{\text {inc }}=45^{\circ}$, which Claussen et al. (2009) obtained by measuring the inclination angle of the $3 \mathrm{D}$ velocity vectors of the $\mathrm{H}_{2} \mathrm{O}$ masers. It is worth mentioning that an independent estimate of the inclination angle of $\sim 40^{\circ}$ was obtained by Sahai et al. (1999) from observations of $\mathrm{OH}$ masers. However, we opted for the value derived by Claussen et al. (2009) because it was obtained in a more straightforward manner. The de-projected length along the major axis of both components was estimated from the maximum separation of the emission peak positions from the reference position (see Fig. 1b), $r_{\max }=1$.' $5 / \cos \theta_{\text {inc }}=2$.' 12 . In this work we adopted a distance to IRAS $16342-3814 D=2 \mathrm{kpc}$ (Sahai et al. 1999). To express the expansion velocity field of the molecular gas we used a power-law function given by the following equation:

$\left[\frac{v_{\exp }(r)}{\mathrm{km} \mathrm{s}^{-1}}\right]=v_{\mathrm{i}}-\left(v_{\mathrm{i}}-v_{\mathrm{f}}\right) \times\left(\frac{r}{r_{\max }}\right)^{\eta}$, where $v_{\mathrm{i}}$ (initial velocity) is the value of the expansion velocity at $r=0, v_{\mathrm{f}}$ (final velocity) is the value of the velocity at the tips of the lobes, and $\eta$ is the exponent of the power-law function.

The values of the initial and final velocity for the HVC can be obtained from the maximum and minimum value of the velocity range of its corresponding kinematical component, that is $150<\left|v_{\text {offset }}\left(\mathrm{km} \mathrm{s}^{-1}\right)\right|<270$ (see Sect. 2). Thus, taking into account the inclination angle, $v_{\mathrm{i}, \mathrm{HVC}}=270 \mathrm{~km} \mathrm{~s}^{-1} / \sin \theta_{\mathrm{inc}}=$ $382 \mathrm{~km} \mathrm{~s}^{-1}$ and $v_{\mathrm{f}, \mathrm{HVC}}=150 \mathrm{~km} \mathrm{~s}^{-1} / \sin \theta_{\text {inc }}=212 \mathrm{~km} \mathrm{~s}^{-1}$. Given that the data points in Fig. 1b exhibit a continuous transition between the two kinematical components, we set the final velocity of the LVC equal the final velocity of the HVC: $v_{\mathrm{f}} \equiv v_{\mathrm{f}, \mathrm{LVC}}=v_{\mathrm{f}, \mathrm{HVC}}$. The initial velocity for the LVC, $v_{\mathrm{i}, \mathrm{LVC}}$, cannot be directly obtained from the observations. From the plot of Fig. $1 \mathrm{~b}$ it can be seen that $v_{\exp }(r=0) \approx 0$, but the points in this plot represent an average position of the distribution of the emission for a certain velocity offset. Therefore, the point located at the origin of coordinates, $(v=0, r=0)$, does not necessarily indicate that $v_{\exp }=0$ when $r=0$, but it means that the average position of the emission is equal to zero in the channel with $v_{\text {offset }}=0$. Consequently, $v_{\mathrm{i}, \mathrm{LVC}}$ was left as a free parameter, together with the exponents of the power-law functions, to be adjusted by further comparison of our model with the observations.

We used the spatio-kinematic modelling tool SHAPE (Steffen et al. 2011) to construct a 3D version of our model of the distribution and kinematics of the molecular gas in IRAS 16342-3814 and compared it with the observations. It should be remarked that this model is not meant to reproduce the emission of any particular molecular line but rather illustrates the overall behaviour of the radiation arising within the kinematical components. First we obtained synthetic images and P-V diagrams of our spatio-kinematical model with SHAPE using initial guess values. Subsequently, we compared the results of SHAPE with the observations in an iterative approach to derive the values of the free parameters of our model (see below). The synthetic P-V diagram obtained with SHAPE and those from the observations were compared qualitatively by eye. 

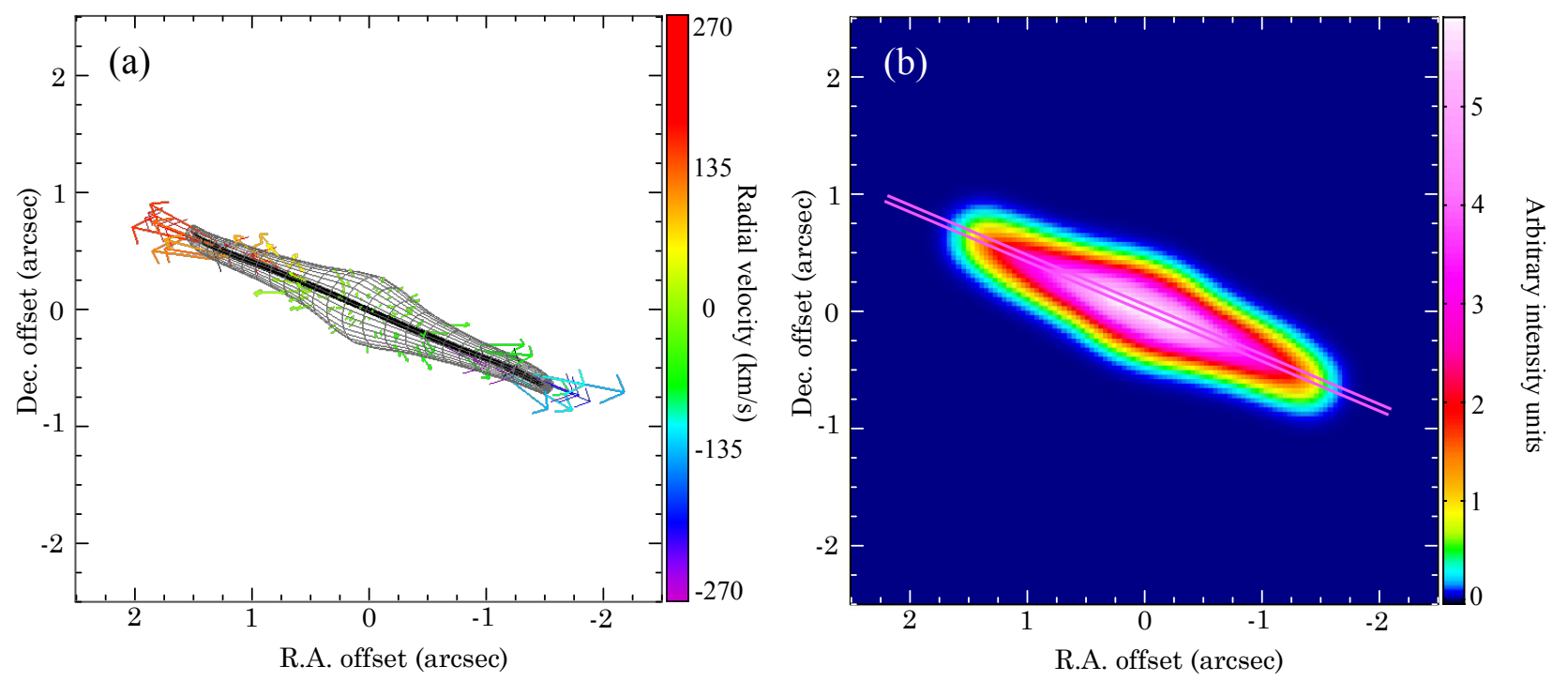

Fig. 4. Panel a: mesh representation of spatial distribution of molecular outflow of IRAS 16342-3814. The HVC and LVC are shown in black and grey colours, respectively. The vectors represent the expansion velocity of the gas and they are coloured according to their component in the line-of-sight. Panel $b$ : brightness distribution of $\mathrm{CO}$ emission obtained from render function of software SHAPE. The intensity is given in arbitrary units. The synthetic image was convolved with a Gaussian function with FWHM (full width half maximum) similar to the synthesised beam of the observations. The parallel lines at $\mathrm{PA}=67^{\circ}$ indicate the direction of a slit used to generate the synthetic P-V diagram shown in Fig. 5a.

After several iterations we found a good fit to the observations using the following parameters for the power-law function: $v_{\mathrm{i}, \mathrm{HVC}}=382 \mathrm{~km} \mathrm{~s}^{-1}, v_{\mathrm{f}, \mathrm{HVC}}=212 \mathrm{~km} \mathrm{~s}^{-1}$, and $\eta_{\mathrm{HVC}}=4.5$ for the HVC; $v_{\mathrm{i}, \mathrm{LVC}}=5 \mathrm{~km} \mathrm{~s}^{-1}, v_{\mathrm{f}, \mathrm{LVC}}=212 \mathrm{~km} \mathrm{~s}^{-1}$, and $\eta_{\mathrm{LVC}}=1.6$ for the LVC. The power-law functions for both components are shown in the plot of Fig. $3 \mathrm{~b}$.

Figure 4a shows a mesh view of the 3D morphology of the emitting region used in SHAPE. We performed a simple radiative transfer of our model using the physical render function of SHAPE, thus the emerging emission is proportional to $T_{\text {ex }}\left(1-e^{-\tau_{v}}\right)$, where $T_{\text {ex }}$ is the excitation temperature and $\tau_{v}$ is the optical depth. The LTE (local thermodynamic equilibrium) option in SHAPE was turned on, which calculates the ratio of the emission coefficient to the absorption coefficient assuming LTE conditions. The absorption coefficient was defined so that $\tau_{v}$ is proportional to the product of the gas density and the length of the emitting region along the line-of-sight, $\tau_{v} \propto n \times l$. For simplicity, the density of the gas and the excitation temperature of the HVC were assumed to be constant throughout the outflow. At the base of the LVC, the excitation temperature was set to a value five times lower than the HVC, increasing monotonically as a function of the distance to reach the same value as the HVC at the tip of the lobes. We note that it is not in the scope of this work to perform a detailed radiative transfer analysis of the model as we are only concerned about the kinematics of the gas. Therefore, the resulting intensity of the synthetic images is given in arbitrary units and do not necessarily correspond to the intensity of the observed emission. The synthetic images were convolved with a Gaussian function with full width half maximum (FWHM) similar to the synthesised beam of the observations.

In Fig. 4b we show a synthetic image of the source emission on the plane of the sky. The spatial distribution of the emission of our model resembles the image of the HVO of IRAS 16342-3814 obtained by Sahai et al. (2017, see their Fig. 2b). The two parallel lines in Fig. $4 \mathrm{~b}$ indicate the direction of a slit used to generate the synthetic P-V diagram shown in Fig. 5a. We used a similar slit to generate a P-V diagram from the data cube of the $\mathrm{CO}(J=3 \rightarrow 2)$ emission, which is shown in Fig. 5b. The faint emission in the upper-right corner of the P-V diagram of Fig. 5b is due to the $\mathrm{H}^{13} \mathrm{CO}(\mathrm{J}=4 \rightarrow 3)$ line overlapping in frequency with the $\mathrm{CO}(J=3 \rightarrow 2)$ emission (see also Fig. 3 of Sahai et al. 2017). The synthetic P-V diagram in Fig. 5a reproduces very well the main features of the $\mathrm{CO}(J=3 \rightarrow 2)$ emission P-V diagram. The clumpy structure that some parts of the synthetic P-V diagram exhibit is due to the binning of the velocity channels during the convolution of the model.

\section{Discussion and interpretation of spatio-kinematical model}

Sahai et al. (2017) proposed a model to explain the P-V diagrams of the $\mathrm{CO}(J=3 \rightarrow 2)$ and $\mathrm{H}^{13} \mathrm{CO}(J=4 \rightarrow 3)$ emission assuming that all the molecular gas is expanding with the same intrinsic velocity. In their model, the S-like pattern seen in the P-V diagram results from the projection effect of the material moving along different directions with respect to the line-of-sight. Under this assumption they concluded that the morphology of the source is an S-like bipolar shape with some gas clouds expanding in directions near the line-of-sight and others expanding near the plane of the sky (see Fig. 3 of Sahai et al. 2017). This model has two main problems: (i) the constant expansion velocity assumption is most likely not true, since the velocity of the gas would depend on the hydrodynamic interaction of the driving jet and the CSE, as Sahai et al. (2017) acknowledge; and (ii) the morphology of the nebula derived from this assumption may result in an S-like shape with a very wide opening angle $\left(\sim 90^{\circ}\right)$ that would not be consistent with the highly collimated morphologies suggested by the IR observations and the $\mathrm{H}_{2} \mathrm{O}$ masers. However, we note that a possible resolution to the problem in (ii) is provided by the Spiral-class PNe introduced by Sahai et al. (2011; e.g. PK 032+07\#2 and PNG 356.8+0.3.3, see their Fig. 7), which, if viewed at a preferred orientation angle, could resemble the lobes seen in IRAS 16342-3814.

In the previous section, Sect. 3, we showed that the spatiokinematical model presented in this work reproduces very well the distribution of the observed $\operatorname{CO}(J=3 \rightarrow 2)$ emission in the 

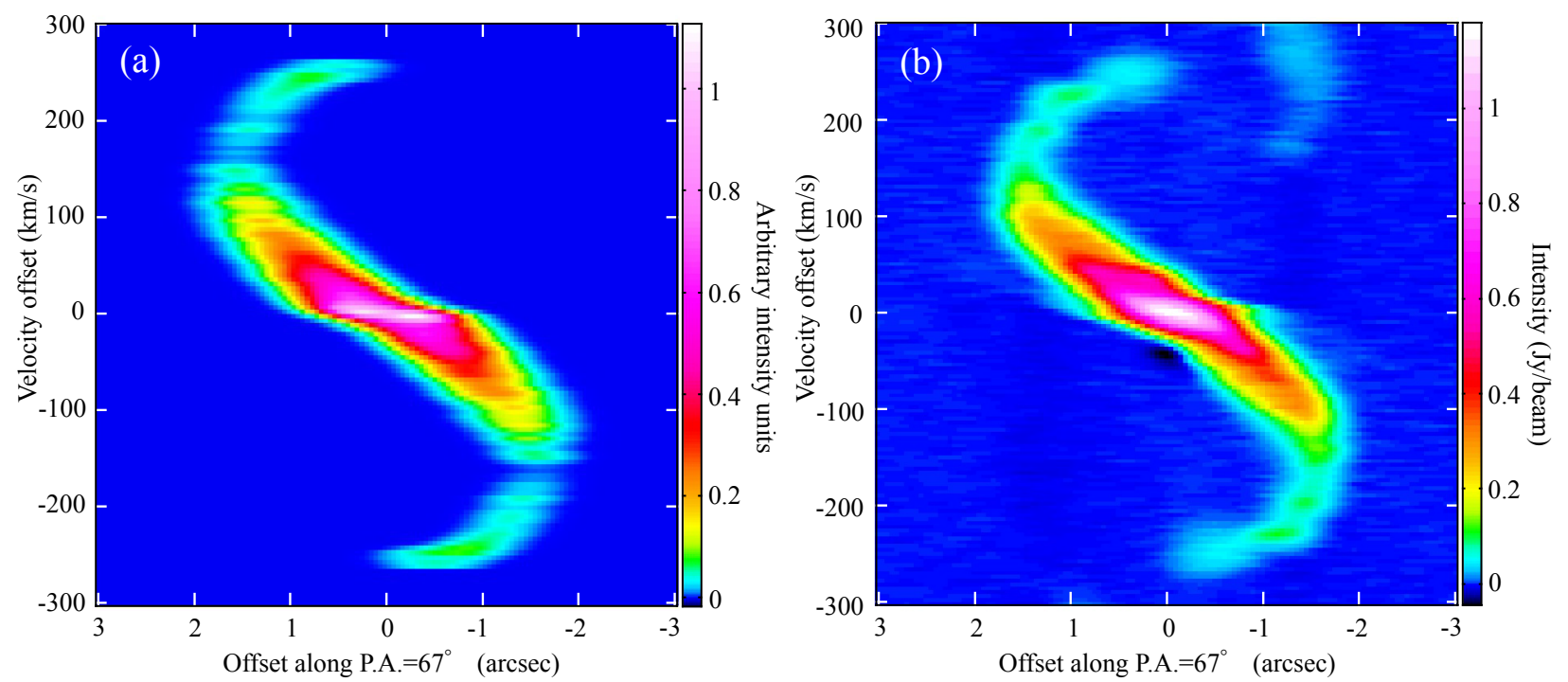

Fig. 5. Panel $a$ : P-V diagram of IRAS 16342-3814 obtained from SHAPE model. Panel $b$ : P-V diagram of IRAS 16342-3814 from ALMA observations. The faint emission in the upper right corner is due to the $\mathrm{H}^{13} \mathrm{CO}(J=4 \rightarrow 3)$ line. Both $\mathrm{P}-\mathrm{V}$ diagrams were obtained using a slit along $\mathrm{PA}=67^{\circ}$.

P-V diagram. As discussed above, the gradients seen in the P-V diagram (Fig. 5b) are interpreted as being due to actual variations of the velocity field of the gas within an elongated narrow region. Therefore, our model circumvents the problem of assuming a constant expansion velocity and reconciles the morphology of the CO gas with the morphology of the source derived from other observations. In the following we discuss the astrophysical origin of the observed velocity gradients. In the past three decades a wealth of observations towards regions of star formation, as well as towards evolved stars, have revealed three main properties that characterise collimated molecular outflows. First, the velocity field of the outflow is described by a proportional relationship between the expansion velocity and the distance to the driving source, meaning $v \propto r^{\eta}$, with $\eta \approx 1$. Second, the collimation of the flow increases systematically with flow velocity and distance from the driving source. And, third, the flow exhibits a power-law variation of mass with velocity, meaning $m(v) \propto v^{-\gamma}$ (Lada \& Fich 1996; Cabrit et al. 1997, and references therein). The most popular mechanism to explain these properties is the jet-driven bow shock (Raga \& Cabrit 1993; Smith et al. 1997). This mechanism naturally creates a positive velocity gradient since as one moves from the broader wings to the narrow apex of the bow shock, the shock becomes less oblique and the net forward velocity increases. In addition, this mechanism produces more swept-up mass at low velocities since the intercepted mass flux is determined by the bow shock cross-section, which steadily grows in the bow wings while the velocity decreases (Cabrit et al. 1997). The LVC of IRAS 16342-3814 exhibits all the properties that characterise jet-driven bow shocks. As shown in Sect. 3, the velocity field of the LVC has a power-law dependence with the distance, although the power-law exponent, $\eta=1.6$, is somewhat larger than the typical values seen in other molecular outflows. The collimation of the LVC increases with velocity as can be seen in Fig. 2. Finally, assuming optically thin emission, LTE conditions, and an average excitation temperature $T_{\mathrm{ex}}=30 \mathrm{~K}$, the mass of gas expanding at a given velocity, that is the mass spectrum $m(v)$, can be obtained from the line profile of the $\mathrm{CO}(J=3 \rightarrow 2)$ emission $^{2}$. In Fig. 6 we show a $\log -\log$ plot of the mass as a function of the velocity

2 The value of the mass estimated in this manner would represent a lower limit of the actual value if the emission is optically thick.

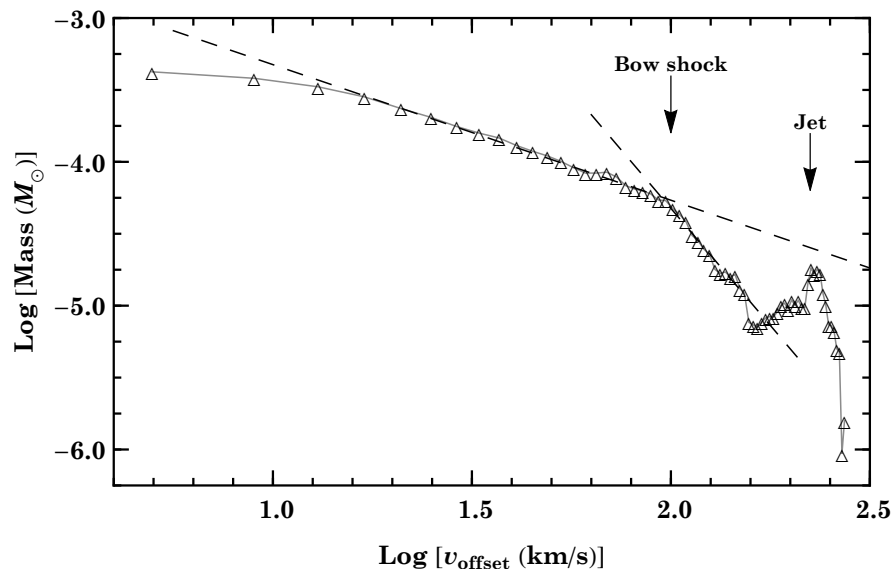

Fig. 6. Molecular mass as a function of expansion velocity obtained from emission blue-shifted with respect to systemic velocity. The dashed lines are power-law fits to the data in the velocity ranges $10<v_{\text {offset }}\left(\mathrm{km} \mathrm{s}^{-1}\right)<100$ and $100<v_{\text {offset }}\left(\mathrm{km} \mathrm{s}^{-1}\right)<150$, respectively. The arrows indicate the emission associated with the bow shock and the jet, respectively.

offset, where we have used a fractional abundance of CO relative to $\mathrm{H}_{2}, f(\mathrm{CO})=3 \times 10^{-4}$ (Sahai et al. 2017). This plot reveals that the emission between $10<v_{\text {offset }}\left(\mathrm{km} \mathrm{s}^{-1}\right)<100 \mathrm{~km} \mathrm{~s}^{-1}$ is described by a power-law $m(v) \propto v^{-\gamma}$ with $\gamma=0.94$, and between $100<v_{\text {offset }}\left(\mathrm{km} \mathrm{s}^{-1}\right)<150 \mathrm{~km} \mathrm{~s}^{-1} \gamma=3$.2. This double power-law variation of the mass with velocity is exactly what observations have revealed in other molecular outflows (Kuiper et al. 1981; Rodriguez et al. 1982; Lada \& Fich 1996), and confirmed by numerical simulations of jet-driven molecular outflows (Smith et al. 1997). However, it should be pointed out that, if the $\mathrm{CO}(J=3 \rightarrow 2)$ line is optically thick, the values of these powerlaw exponents simply represent a lower limit. Furthermore, it cannot be ruled out that the break of the power-law exponent around $v_{\text {offset }}=100 \mathrm{~km} \mathrm{~s}^{-1}$ might be due to a change in the optical depth regime.

In Fig. 7 we show contours indicating the $\operatorname{CO}(J=1 \rightarrow 0)$ emission superimposed on a moment- 8 (maximum value of the spectrum for each pixel) image of the $\mathrm{CO}(J=3 \rightarrow 2)$ emission. The $\mathrm{CO}(J=1 \rightarrow 0)$ emission was averaged over the velocity 


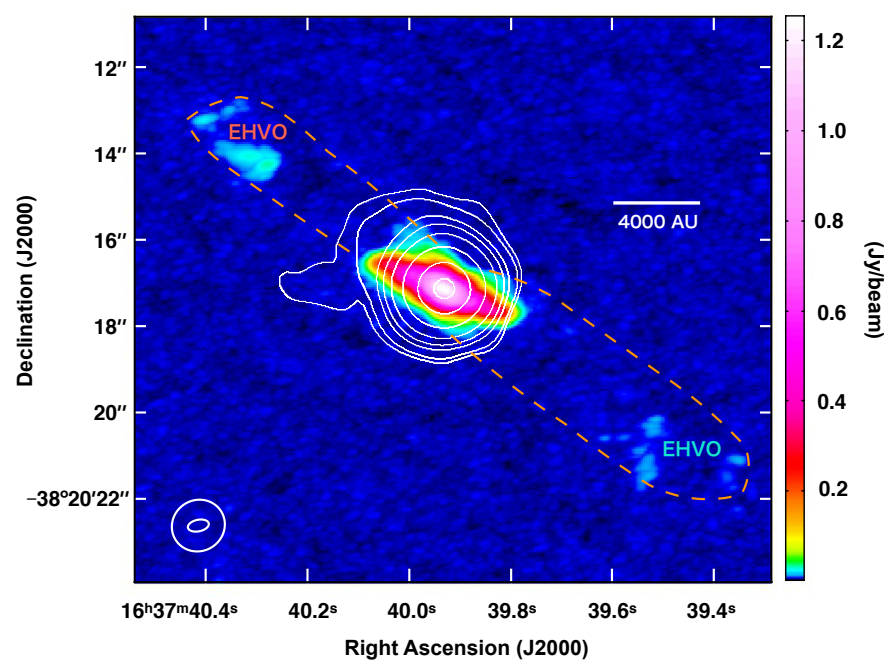

Fig. 7. CO emission from IRAS 16342-3814. The colour scale corresponds to the moment- 8 (maximum value of the spectrum for each pixel) image of the $\mathrm{CO}(J=3 \rightarrow 2)$ emission. The contours indicate the $\operatorname{CO}(J=1 \rightarrow 0)$ emission averaged over the velocity range $-10<v_{\text {offset }}\left(\mathrm{km} \mathrm{s}^{-1}\right)<+10$ with values $(0.007,0.01,0.02,0.03,0.05$, $0.1,0.2,0.3) \mathrm{Jy}_{\text {beam }}^{-1}$, where the first contour represents four times the value of the rms. The orange dashed line delineates the region that contains emission from the EHVO. The ellipses located at the lower left corner indicate the synthesised beams of the $\mathrm{CO}(J=3 \rightarrow 2)$ and $\operatorname{CO}(J=1 \rightarrow 0)$ observations, respectively. The beam size of the $\mathrm{CO}(J=3 \rightarrow 2)$ observations is the same as in Fig. 2 and the beam size of the $\mathrm{CO}(J=1 \rightarrow 0)$ observations is 1 ". $^{\prime} 25 \times 1$ !' $^{\prime \prime} 18$ with $\mathrm{PA}=-58^{\circ}$.

range $-10<v_{\text {offset }}\left(\mathrm{km} \mathrm{s}^{-1}\right)<+10$, which is the typical expansion velocity of the CSE formed during the AGB phase. The brightness distribution of the emission is round and has a deconvolved size of $\sim 00^{\prime \prime} 9$, which is almost twice as large as the deconvolved size of the $\operatorname{CO}(J=3 \rightarrow 2)$ emission in the same velocity range (see Sect. 3), suggesting that it is tracing material of the CSE created by the AGB wind. The existence of a relic AGB circumstellar envelope is also suggested by the lowvelocity $\mathrm{OH} 1612$ maser emission, which exhibits maser features distributed over a region more extended than the bipolar outflow (see Fig. 1 of Sahai et al. 1999). Moreover, Murakawa \& Izumiura (2012) showed that the spectral energy distribution (SED) of IRAS 16342-3814 is best reproduced by a model that includes a disc, a torus, bipolar lobes, as well as a spherical AGB envelope. Thus, given these arguments, together with the results presented in the previous paragraph, we conclude that the LVC in IRAS 16342-3814 corresponds to material of the CSE that has been swept-up by the jet-driven bow shock.

A feature revealed in these ALMA observations that is not commonly found as notably as in other molecular outflows, is the emission associated with the HVC. Figure 6 shows that for velocities offsets higher than $150 \mathrm{~km} \mathrm{~s}^{-1}$ the mass spectrum exhibits a plateau followed by a sharp peak. Smith et al. (1997) obtained a similar feature in the intensity profile from their hydrodynamic simulations of a jet-driven outflow. They identified this feature with the jet's direct emission (see Figs. 1 and 2 of Smith et al. 1997). The deceleration of these components suggests the presence of turbulent mixing through Kelvin-Helmholtz instabilities at the velocity shear between the jet and the ambient gas. If turbulent entrainment along the beam is the dominant process, then the jet decreases in average velocity along its length as it transfers momentum to the entrained material (Chernin et al. 1994). Given a velocity of the jet $v_{\exp }=380 \mathrm{~km} \mathrm{~s}^{-1}$, a sound speed in the medium $c_{\mathrm{s}} \sim 1 \mathrm{~km} \mathrm{~s}^{-1}$, a density of the medium $n_{\mathrm{H}_{2}}=10^{6} \mathrm{~cm}^{-3}$, and a kinetic temperature $T_{\mathrm{k}} \sim 100 \mathrm{~K}$, the Reynolds number of the jet is $\mathcal{R} e_{\mathrm{m}}>10^{10}$. Consequently, the jet is expected to be highly turbulent and suffer deceleration, which is indeed what the observations reveal. We conclude that the HVC represents material entrained by the underlying jet near its axis or it could be the molecular component of the jet itself.

As mentioned earlier, molecular outflows with the characteristic properties of a jet-driven outflow have also been found in the CSE of some evolved stars (e.g., Bujarrabal et al. 1998; Sánchez Contreras et al. 2000; Alcolea et al. 2001, 2007). For these objects, the linear relationship between the expansion velocity and the distance to the driving source has been interpreted in terms of free expansion of the gas after having suffered a strong axial acceleration by a collimated wind or jet during a time period much shorter than the whole post-AGB lifetime of the source (Bujarrabal et al. 1998; Alcolea et al. 2001). Particularly, Alcolea et al. (2001) derived an upper limit to the duration of this post-AGB interaction in the pre-planetary nebula $\mathrm{OH} 231.8+4.2$ of $\sim 125 \mathrm{yr}$. Our results suggest that in IRAS 16342-3814 we are witnessing the presence of two different processes of entrainment, one called "prompt entrainment", which transfers momentum through the leading bow shock, and the second is "steady-state entrainment", which refers to ambient gas that is entrained along the sides of the jet (De Young 1986; Chernin et al. 1994). The former would lead to the formation of the linear relationship of the velocity with the distance seen in the PV diagrams of the molecular emission of the more evolved objects, which corresponds to swept-up material in a jet-driven bow shock. The new ingredient revealed in these ALMA observations is the HVC, which is related to the driving jet. It is likely that in the molecular outflows seen in other more evolved objects the jet has long disappeared as it is expected to last only for a couple hundred years. This strengthens the idea that wf-nebulae are indeed undergoing the ephemeral transition where they develop the collimated outflows that shape the CSE leading to the formation of asymmetrical PNe. In fact, recently Orosz et al. (2018) showed a superb example of a decelerating outflow in the wfnebula IRAS $18113-2503$ traced by $\mathrm{H}_{2} \mathrm{O}$ masers, which is likely to be directly related to the driving jet.

\subsection{Molecular jet and equatorial waist timescale}

The plot on Fig. $3 \mathrm{~b}$ and the P-V diagram in Fig. 5b show an instantaneous picture of the velocity field in the outflow at present time. Thus they cannot be used to trace the velocity history of the individual gas particles, unless the deceleration law as a function of time is known. In order to understand the evolution of the P-V diagram as a function of time an entire understanding of the complex dynamical system of the outflow is required. Consequently, it is difficult to estimate the exact lifetime of the outflow. However, if we make the reasonable assumption that the jet has been launched at roughly the same speed throughout its lifetime, it is clear that the gas particles located at the head of the jet suffered a deceleration from the initial velocity, $v_{\mathrm{i}}$, to the final velocity, $v_{\mathrm{f}}$, across its length, $r_{\max }$. If we consider a constant deceleration function, the lifetime of the outflow can be obtained as $\tau_{\text {jet }}=2 \times r_{\text {max }} /\left(v_{\mathrm{i}}+v_{\mathrm{f}}\right) \approx 70 \mathrm{yr}$. This value is simply a lower limit for the lifetime as it is likely that the jet suffered a steeper deceleration in a region closer to the star, where the density of the CSE is larger. For example, if the head of the jet decelerated to its current value, $v_{\mathrm{f}}$, soon after it was launched and it has been expanding at nearly constant velocity afterwards, the lifetime of the jet is $\tau_{\text {jet }}=r_{\text {max }} / v_{\mathrm{f}} \approx 100 \mathrm{yr}$.

Sahai et al. (2017) identified a toroidal structure in IRAS $16342-3814$ from the emission of the $\mathrm{H}^{13} \mathrm{CO}(4 \rightarrow 3)$ line 
and derived a radius $r_{\text {torus }} \approx 0$ ". 33 and an expansion velocity of $v_{\text {torus }}=20 \mathrm{~km} \mathrm{~s}^{-1}$, which gives an expansion timescale of the torus $\tau_{\text {torus }} \approx 160 \mathrm{yr}$. This value is larger than the timescale of the jet derived above. However, this timescale is obtained assuming that the gas of the torus expanded at constant velocity. According to our interpretation of the LVC (see Sect. 4), this toroidal structure would correspond to shocked gas in the equatorial region expanding perpendicularly to the jet (see e.g., Soker \& Rappaport 2000). It is expected that the velocity of the shock would decrease as it interacts with the ambient gas of the CSE. Therefore, the value derived assuming a constant expansion velocity would simply be an upper limit of the lifetime of the torus. As mentioned earlier, a complete understanding of the acceleration history of the gas is necessary to calculate the exact lifetime of the torus. In the case of a jet-driven bow shock the lifetimes of the toroidal structure and the jet should be the same.

It is worth noting that the location of the $\mathrm{H}_{2} \mathrm{O}$ masers observed by Claussen et al. (2009) in the P-V diagram corresponds to emission from the LVC of the molecular gas. Therefore, it is unlikely that they are tracing the bow shock at the tip of the jet but rather they seem to be associated with the bow shock expanding laterally. This could explain the observed cessation of expansion of the $\mathrm{H}_{2} \mathrm{O}$ masers in this source (Rogers et al. 2012), although this could also be due to the masers having reached the edge of the dense region of the CSE, as suggested by Fig. 7. Given this, we emphasise that the lifetime of the jet, hence the lifetime of the whole molecular outflow, cannot be obtained directly from the kinematical timescale of the $\mathrm{H}_{2} \mathrm{O}$ masers. It is reasonable to expect that this is also true for other wf-nebulae, as discussed by Yung et al. (2017).

\subsection{The nature of the EHVO}

The molecular outflow considered in this work (which in the nomenclature of Sahai et al. (2017) is also referred to as HVO) and the EHVO exhibit significant differences in their intrinsic properties. Firstly, as pointed out by Sahai et al. (2017), there is an angle of $\sim 12^{\circ}$ between the directions of the main axes of the EHVO and the HVO (see Figs. 1 and 7 of Sahai et al. 2017). Secondly, considering the apparent extension of the EHVO on the plane of the sky $\left(\sim 14^{\prime \prime}\right)$, the projected radial velocity ( $\sim 310 \mathrm{~km} \mathrm{~s}^{-1}$; Sahai et al. 2017), and assuming constant velocity, the estimated kinematical timescale is $\tau_{\mathrm{EHVO}} \sim 215 \times \tan \theta_{\mathrm{EHVO}} \mathrm{yr}$, where $\theta_{\text {EHVO }}$ is the inclination of the EHVO with respect to the plane of the sky. This timescale is larger than the one derived in the previous subsection for the HVO, although the actual value depends on the inclination, as well as on the initial velocity and the deceleration of the EHVO. Finally, the EHVO exhibits an expansion velocity that does not correspond to the velocity expected from the kinematical model presented in this work. We thus conclude that the clumps of the EHVO seen in Fig. 7 correspond to material that was swept up by a different outflow, which is faster and likely older than the HVO. Additionally, there seems to be traces of entrained material of the EHVO outflow that appear as "horns" of the HVO and confined within the region traced by the $\operatorname{CO}(J=1 \rightarrow 0)$ emission. Therefore, it is likely that the extension of the region traced by the $\operatorname{CO}(J=1 \rightarrow 0)$ emission determines the radius of $\mathrm{CO}$ photo-dissociation by interstellar $\mathrm{UV}$ radiation.

\subsection{Kinetic energy and scalar momentum of molecular outflow}

Since our model is fundamentally different from the one presented by Sahai et al. (2017), here we re-calculate the scalar momentum and kinematic energy of the molecular outflow. To obtain the kinetic energy and scalar momentum, in principle, it is possible to use the mass spectrum shown in Fig. 6. However, the velocity offset in this plot is simply the component of the expansion velocity on the line-of-sight. The 3D expansion velocity is necessary for the calculations. To circumvent this problem, one can take advantage of the morphology adopted for the emitting region, which has an elongated, narrow shape. In such a case, it is possible to de-project the velocity vectors by multiplying the velocity offsets by a factor of $1 / \sin \theta_{\text {inc }}$, with $\theta_{\text {inc }}=45^{\circ}$. This is not strictly true for all the velocity vectors, since the gas is not really moving along one single line. Particularly, for the gas located close to the central source, which might be expanding along the line-of-sight, the expansion velocity will be overestimated by a factor of $1 / \sin \theta_{\text {inc }}$. For such gas, this leads to an overestimation of the scalar momentum by a factor of $\sqrt{2}$ and for the kinetic energy by a factor of 2 . In addition, there is an uncertainty due to the optical depth of the $\mathrm{CO}(J=3 \rightarrow 2)$ emission since it was assumed to be optically thin to obtain the mass spectrum of Fig. 6. If the emission is optically thick the estimated value of the mass simply represents a lower limit. Having this in mind, we proceeded to calculate the kinetic energy and scalar momentum in the manner introduced by Bujarrabal et al. (2001) and described in the following. Firstly, we multiplied the mass in each channel by the square of the corresponding velocity to obtain the kinetic energy per channel. By adding the kinetic energy of all the channels we obtain $K_{\mathrm{LVC}}=8 \times 10^{44} \mathrm{erg}$ and $K_{\mathrm{HVC}}=1 \times 10^{45} \mathrm{erg}$ for the LVC and $\mathrm{HVC}$, respectively. Similarly, the derived scalar momentum is $1.6 \times 10^{38} \mathrm{gm} \mathrm{cm} \mathrm{s}^{-1}$ and $6 \times 10^{37} \mathrm{gm} \mathrm{cm} \mathrm{s}^{-1}$ for the LVC and HVC, respectively. The total kinetic energy and scalar momentum in the outflow are of the same order of magnitude as the values calculated by Sahai et al. (2017). Subsequently, the scalar momentum in the molecular outflow transferred by the jet, $\Delta P$, is obtained by subtracting the scalar momentum due to the AGB wind from the total scalar momentum: $\Delta P=\left(P_{\mathrm{LVC}}+\right.$ $\left.P_{\mathrm{HVC}}\right)-\left(M_{\text {outflow }} \times v_{\mathrm{AGB}}\right)$, where $P_{\mathrm{LVC}}$ and $P_{\mathrm{LVC}}$ are the scalar momentum of the LVC and HVC, respectively; $v_{\mathrm{AGB}}$ is the expansion velocity of the AGB wind and $M_{\text {outflow }}$ is the total mass of the outflow. From the mass spectrum in Fig. 6 we estimate a total molecular mass in the outflow $M_{\text {outflow }}=1.6 \times 10^{-2} M_{\odot}$, thus $\Delta P=1.6 \times 10^{38} \mathrm{gm} \mathrm{cm} \mathrm{s}{ }^{-1}$, where we have assumed an expansion velocity of the AGB wind $v_{\text {AGB }} \sim 20 \mathrm{~km} \mathrm{~s}^{-1}$. As pointed out by Sahai et al. (2017), given a star luminosity of $L_{\star}=6000 L_{\odot}$ for IRAS $16342-3814$, the radiation alone cannot account for the scalar momentum of the molecular outflow. The mass-loss rate of the driving jet can be estimated by considering the extreme case in which the kinetic energy of the jet is purely transformed into kinetic energy of the molecular outflow: $\dot{M}_{\text {jet }}=\left[K_{\mathrm{LVC}}+K_{\mathrm{HVC}}-0.5\left(M_{\text {outflow }} \times v_{\mathrm{AGB}}^{2}\right)\right] /\left(v_{\text {jet }}^{2} \tau_{\text {jet }}\right)$, where $K_{\mathrm{LVC}}$ and $K_{\mathrm{LVC}}$ are the kinetic energies of the LVC and HVC, respectively, and the kinetic energy due to the AGB wind has been subtracted. Using $v_{\text {jet }}=380 \mathrm{~km} \mathrm{~s}^{-1}$ and $\tau_{\text {jet }}=100 \mathrm{yr}$, the resulting mass-loss rate of the primary jet, meaning the one driving the molecular outflow, is $\dot{M}_{\text {jet }}=5 \times 10^{-6} M_{\odot} \mathrm{yr}^{-1}$. As a matter of fact, it is known that part of the energy transferred by the jet is radiated away, for example as $\mathrm{H}_{2}$ emission (Gledhill \& Forde 2012), thus this value simply represents a lower limit for the mass-loss rate of the jet.

\subsection{The precessing jet in IRAS 16342-3814}

The distribution of the emission peak positions of the highvelocity emission in Fig. 1a hints at an oscillating pattern with 


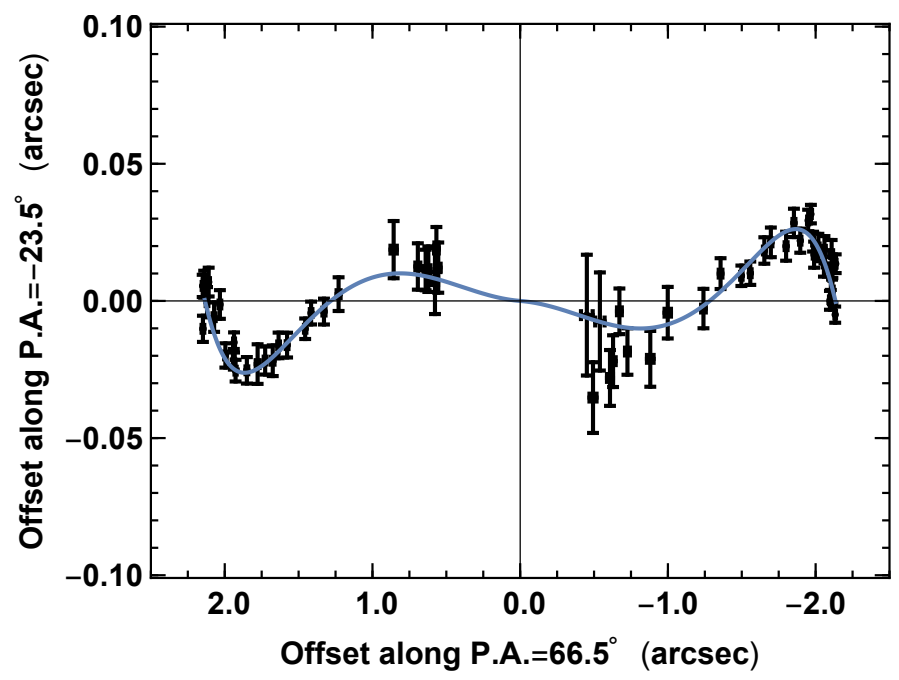

Fig. 8. Oscillating pattern of positions of peak-emission for highvelocity emission. The blue line indicates a sinusoidal function fit to the data using Eq. (2).

respect to the direction of the major axis of the structure. To further investigate the distribution of the peak positions of the high-velocity emission we rotated the data points by an angle of $\theta_{\text {rot }} \approx 23^{\circ}$ and plotted them as shown in Fig. 8. From this plot it can be seen that the pattern of the peak positions exhibits an oscillating point-symmetric pattern.

This pattern can be explained by the presence of a collimated outflow that precesses around a fixed axis. From Fig. 8 it can be seen that the amplitude of the oscillations is much smaller than the extent of the pattern, thus implying a very small precessing angle. If we assume that every particle of the jet has decelerated at a constant rate, $a_{\mathrm{n}}=\left[v\left(r_{\mathrm{n}}\right)^{2}-v_{\mathrm{i}}^{2}\right] /\left(2 r_{\mathrm{n}}\right)$, during its journey from the base of the outflow to the radius $r=r_{\mathrm{n}}$, it is possible to express the oscillating pattern of the emission peak positions with a sinusoidal function expressed as:

$y(r)=A \times r \times \sin \left[\frac{2 \pi \tau(r)}{T}-\phi\right]$,

where $\tau(r)$ is the travelling-time of the particle from the base of the outflow to the radius $r$ and it is given by

$\tau(r)=-v_{\mathrm{i}}+\frac{\left[v_{\mathrm{i}}^{2}+2 r a(r)\right]^{1 / 2}}{a(r)}$,

where $v_{\mathrm{i}}$ is the initial velocity of the jet and $a(r)$ is the acceleration of the particle of the jet located at a distance $r$. The factor $A \times r$ in Eq. (2) expresses the amplitude of the sinusoidal pattern as a function of $r$. Thus, the parameter $A$ is the tangent of the semi-opening angle of the pattern. $T$ is the precession period and $\phi$ is the phase of the sinusoidal pattern.

We fitted the precession model given by Eq. (2) to the data points of the pattern seen in Fig. 8. However, the shape of the pattern depends on the angle, $\theta_{\text {rot }}$, used for rotating the positions of the data points. Therefore, $\theta_{\text {rot }}$ is also a free parameter. Considering the PA of the line connecting the $\mathrm{H}_{2} \mathrm{O}$ masers detected by Claussen et al. (2009), the rotation angle is $\theta_{\text {rot }}=23.9$ and the best fit parameters are $A=0.018 \pm 0.001$, $T=86 \pm 4 \mathrm{yr}, \phi=57 \pm 10^{\circ}$. If we adopt the PA of the nebula's long axis determined by Sahai et al. (2017), the rotation angle is $\theta_{\text {rot }}=23^{\circ}$, which results in the following best fit parameters:
$A=0.010 \pm 0.001, T=58 \pm 5 \mathrm{yr}$, and $\phi=29 \pm 30^{\circ}$. In Fig. 8 we plot the best fit function for an intermediate value of the rotation angle $\theta_{\text {rot }}=23.5$. The blue line in the plot shows the fit to the data with parameters, $A=0.014 \pm 0.001, T=72 \pm 4 \mathrm{yr}$, $\phi=-17 \pm 16^{\circ}$. The corresponding opening angle of the pattern can be obtained as $2 \times \operatorname{atan}(A)=\theta_{\mathrm{op}} \approx 2^{\circ}$. Given the limited angular and spectral resolution of these observations, it is difficult to discern which rotation angle gives the best fit. Higher angular and spectral resolution observations are necessary to unambiguously identify the symmetry axis of the jet and better constrain its parameters.

The precession period derived for the sinusoidal pattern of the CO-emission peak positions is slightly longer than the one obtained for the corkscrew pattern found by Sahai et al. (2005) from IR observations, $T \lesssim 50 \mathrm{yr}$, even considering the uncertainty in the rotation angle. The period of the corkscrew pattern was obtained assuming that the velocity of the dust is similar to the velocity of the $\mathrm{OH}$ masers, $v_{\mathrm{OH}} \sim 90 \mathrm{~km} \mathrm{~s}^{-1}$. However, under the action of an external force, such as the one exerted by the ram pressure of the driving jet, the velocity of the dust could be lower than the velocity of the gas, since the inertial mass of the dust grains is larger than that of the gas particles. Indeed, Yoshida et al. (2011) found that the dust in the superwind of the starburst galaxy M 82 is kinematically decoupled from the gas, with the former moving substantially slower than the latter. In such a case, the value obtained by Sahai et al. (2005) would represent a lower limit for the period. For example, if one takes an expansion velocity of $v_{\exp }=50 \mathrm{~km} \mathrm{~s}^{-1}$ for the dust, the period of the corkscrew pattern is $\sim 70 \mathrm{yr}$, which is within the range of the values obtained for the $\mathrm{CO}$ pattern. We propose that the sinusoidal pattern seen in Fig. 8 traces the underlying outflow that drives the dust outwards and forms the corkscrew pattern seen in the IR.

\section{Conclusions}

In this work we analyse the $\mathrm{CO}(J=3 \rightarrow 2)$ emission of the wfnebula IRAS 16342-3814 to further study the spatio-kinematical characteristics of the molecular gas. We identified two components with different kinematical characteristics: one that exhibits deceleration, and the other with velocity increasing as a function of distance. We propose a spatio-kinematical model that includes a decelerating collimated component surrounded by more extended component with a positive velocity gradient. The elongated narrow morphology of the model is in good agreement with results from previous IR and $\mathrm{H}_{2} \mathrm{O}$ maser observations. We created synthetic images and P-V diagrams using the morphology and velocity fields of our model. The resulting synthetic images and P-V diagrams reproduce the observations very well. The velocity field (together with the mass spectrum derived from the emission) and morphology of the emitting region are consistent with a jet-driven molecular outflow. The decelerating component is associated with material entrained by the underlying jet near its axis or it could be the molecular component of the jet itself. This feature is not seen in other more evolved objects that exhibit more developed bipolar morphologies. We conclude it to be likely that the jet in those objects has already disappeared as it is expected to last only a couple hundred years. This strengthens the idea that wf-nebulae are undergoing a very short transition where they develop the collimated outflows that shape the CSE. The timescale for the molecular outflow is $\tau_{\text {jet }} \sim 70$ $100 \mathrm{yr}$. The scalar momentum carried by the molecular outflow is much larger than can be provided by the radiation of the central star during the short lifetime of the outflow. We identify an 
oscillating point-symmetric pattern for the component. We fitted the data points using a sinusoidal function and derived a period of $T \sim 60-90 \mathrm{yr}$ and an opening angle of $\theta_{\mathrm{op}} \approx 2^{\circ}$. We propose that this pattern traces the jet that drives the material that forms the corkscrew pattern seen in the IR.

For the first time ALMA is allowing the full and detailed study of the morphology of collimated molecular outflows underlying the high-velocity structures traced by $\mathrm{H}_{2} \mathrm{O}$ maser in wf-nebulae. A follow-up study of these objects with ALMA would be of great importance for understanding the launching, collimation, and interaction with the circumstellar material of the collimated outflows of wf-nebuale.

Acknowledgements. This paper makes use of the following ALMA data ADS/JAO.ALMA\#2012.1.00678.S. ALMA is a partnership of ESO (representing its member states), NSF (USA), and NINS (Japan), together with NRC (Canada), MOST and ASIAA (Taiwan), and KASI (Republic of Korea), in cooperation with the Republic of Chile. The Joint ALMA Observatory is operated by ESO, AUI/NRAO, and NAOJ. DT was supported by the ERC consolidator grant 614264. GO would like to acknowledge financial support from the NSFC (Grant No. 11503072), the National Key R\&D Program of China (Grant No. 2018YFA0404602), and the Youth Innovation Promotion Association of the CAS. The authors thank Susanne Aalto for helpful discussions. The authors also thank the anonymous referee for constructive comments and suggestions that helped to improve the manuscript.

\section{References}

Alcolea, J., Bujarrabal, V., Sánchez Contreras, C., Neri, R., \& Zweigle, J. 2001 , A\&A, 373, 932

Alcolea, J., Neri, R., \& Bujarrabal, V. 2007, A\&A, 468, L41

Bujarrabal, V., Alcolea, J., \& Neri, R. 1998, ApJ, 504, 915
Bujarrabal, V., Castro-Carrizo, A., Alcolea, J., \& Sánchez Contreras, C. 2001, A\&A, 377, 868

Cabrit, S., Raga, A., \& Gueth, F. 1997, in Herbig-Haro Flows and the Birth of Stars, eds. B. Reipurth \& C. Bertout, IAU Symp., 182, 163

Chernin, L., Masson, C., Gouveia dal Pino, E. M., \& Benz, W. 1994, ApJ, 426, 204

Claussen, M. J., Sahai, R., \& Morris, M. R. 2009, ApJ, 691, 219

De Young, D. S. 1986, ApJ, 307, 62

Gledhill, T. M., \& Forde, K. P. 2012, MNRAS, 421, 346

Imai, H. 2007, in Astrophysical Masers and their Environments, eds. J. M. Chapman \& W. A. Baan, IAU Symp., 242, 279

Imai, H., Chong, S. N., He, J.-H., et al. 2012, PASJ, 64, 98

Kuiper, T. B. H., Zuckerman, B., \& Rodriguez-Kuiper, E. N. 1981, ApJ, 251, 88

Lada, C. J., \& Fich, M. 1996, ApJ, 459, 638

Likkel, L., \& Morris, M. 1988, ApJ, 329, 914

Murakawa, K., \& Izumiura, H. 2012, A\&A, 544, A58

Raga, A., \& Cabrit, S. 1993, A\&A, 278, 267

Rodriguez, L. F., Carral, P., Ho, P. T. P., \& Moran, J. M. 1982, ApJ, 260, 635

Rogers, H., Claussen, M., Morris, M. R., \& Sahai, R. 2012, Am. Astron. Soc. Meeting Abstracts, 219, 348

Sahai, R., \& Trauger, J. T. 1998, AJ, 116, 1357

Sahai, R., te Lintel Hekkert, P., Morris, M., Zijlstra, A., \& Likkel, L. 1999, ApJ, 514, L115

Sahai, R., Le Mignant, D., Sánchez Contreras, C., Campbell, R. D., \& Chaffee, F. H. 2005, ApJ, 622, L53

Sahai, R., Morris, M. R., \& Villar, G. G. 2011, AJ, 141, 134

Sahai, R., Vlemmings, W. H. T., Gledhill, T., et al. 2017, ApJ, 835, L13

Smith, M. D., Suttner, G., \& Yorke, H. W. 1997, A\&A, 323, 223

Soker, N., \& Rappaport, S. 2000, ApJ, 538, 241

Steffen, W., Koning, N., Wenger, S., Morisset, C., \& Magnor, M. 2011, IEEE Trans. Vis. Comput. Graphics, 17, 454

Sánchez Contreras, C., Bujarrabal, V., Neri, R., \& Alcolea, J. 2000, A\&A, 357, 651

Yoshida, M., Kawabata, K. S., \& Ohyama, Y. 2011, PASJ, 63, 493

Yung, B. H. K., Nakashima, J.-i., Hsia, C.-H., \& Imai, H. 2017, MNRAS, 465, 4482 